\title{
Pancreaticoduodenectomy and Vascular Resection: Persistent Controversy and Current Recommendations
}

\author{
Kathleen Christians, MD and Douglas B. Evans, MD \\ Department of Surgery, The Medical College of Wisconsin, Milwaukee, WI
}

In this issue of the Journal, Giovanni and colleagues analyze 12 recent manuscripts which reported the outcome of patients with pancreatic cancer who required portal vein (PV) or superior mesenteric vein (SMV) resection at the time of pancreaticoduodenectomy (PD). ${ }^{1}$ They conclude that venous resection can be safely performed and should be considered in appropriately selected patients. Fortner first popularized vascular resection at the time of PD in 1973 when he proposed the use of "regional pancreatectomy." 2 However, this concept has remained controversial for the following reasons:

1. Most physicians do not understand the difference between the historical experience with regional pancreatectomy and isolated tumor resection of the SMV, PV or superior mesenteric-portal vein (SMPV) confluence performed as part of a gross complete resection of the primary tumor. Vascular resection at the time of PD was initially performed in an attempt to improve survival duration by performing an en bloc resection of the pancreas and surrounding structures. ${ }^{2}$ This concept of regional pancreatectomy involved the systematic resection of major peripancreatic vascular structures together with wide soft tissue clearance. Contrary to the beliefs of Fortner and others, radical PD has not been demonstrated to confer a survival benefit. ${ }^{3,4}$ Most physicians and many surgeons assume that the negative experience with regional pancreatectomy also applies to patients with isolated tumor extension that involves a short segment of the SMV or PV.

2. The addition of vascular resection and reconstruction to PD increases the complexity of the operation and is

(C) Society of Surgical Oncology 2009

Published Online: 24 January 2009

K. Christians, MD

e-mail: kchristi@mcw.edu not something that all pancreatic surgeons feel capable of performing. This issue may be amplified by the limited experience of many surgeons with the technical aspects of vascular surgery and the potential for perioperative death and major morbidity that exists with pancreatic surgery for cancer.

3. The published data which examines vascular resection as a prognostic factor for survival duration is of poor quality. The majority of such reports (including those analyzed by Giovanni and colleagues) did not contain a description of the process used to differentiate complete $(\mathrm{R} 0 / \mathrm{R} 1)$ from incomplete (R2) gross resections. Even in those patients who may have undergone a complete gross resection, most manuscripts failed to incorporate prospective standardized pathologic evaluation and reporting of the $\mathrm{PD}$ specimen to differentiate $\mathrm{R} 0$ from $\mathrm{R} 1$ resections. This is a critically important consideration because the intraoperative finding of venous adherence to the tumor is often unexpected and the surgeon may then attempt to separate the SMPV confluence from the pancreatic head. When this maneuver is unsuccessful, the surgeon is left with either a grossly positive margin or an inadvertent venotomy. Venous injury often results in uncontrolled hemorrhage and the necessity for rapid removal of the tumor without proper attention to the SMA dissection; it is easy to appreciate how such cases may result in an $\mathrm{R} 2$ resection. In patients with grossly incomplete resections, and without any form of preoperative therapy, it is likely that their poor survival is due to the persistent adenocarcinoma at the SMA or celiac origin, not the presence of a vascular reconstruction. In the absence of prospective evaluation of the SMA margin (performed by very few of the papers analyzed by Giovanni and colleagues), reports of venous resection during PD are impossible to interpret. Further, even if the SMA margin is assessed accurately, the pathologist cannot differentiate an R2 
from an R1 resection; the surgeon's operative note must state whether or not a gross complete resection was performed..$^{5}$

What we do know about venous resection at the time of PD:

1. There should be no biologic significance to tumor abutment or invasion of the lateral wall of the SMPV confluence (in comparison with tumor invasion of second- or third-order branches of the SMV) as the primary tumor has access to the systemic circulation very early in the disease course before diagnosis. ${ }^{6,7}$ Such tumor abutment or invasion of the wall of the SMV occurs due to continued growth of the primary tumor and is a reflection of both the location of the tumor within the pancreatic head and tumor size. This occurs long after the primary tumor has had access to the systemic circulation. In addition, the biologic process of metastatic growth in distant organs is a complex event more dependent on the genetic profile of the primary tumor (and the host) rather than its size or location within the pancreas.

2. The SMV and PV are not surrounded by nerve tissue. In contrast, the visceral arteries including the celiac axis, hepatic artery, and SMA are surrounded by the autonomic nerves which innervate the gastrointestinal tract. Pancreatic cancers are frequently characterized by extrapancreatic infiltrative growth, perineural invasion, and extension along the autonomic nerves. One can often see tumor extension along the SMA or celiac axis extending posteriorly to the aorta and this neural invasion is thought responsible for the pain associated with this disease. In such cases, resection of the involved artery, if performed, may not clear the posterior extent of disease, which may involve the para-aortic autonomic ganglia. The visceral veins such as the SMV or PV do not have an investing sheath of autonomic neural tissue.

3. Circumferential skeletonization or segmental resection of the SMA will deinnervate the small bowel and frequently result in rapid gastrointestinal transit. In patients of normal or thin body habitus this will cause nutritional depletion and the need for total parenteral nutrition. Such is not the case in patients who require segmental resection of the SMV or PV.

4. In contrast to tumor-artery abutment, which can be accurately interpreted on good-quality computed tomography (CT) imaging, tumor abutment of the lateral or posterolateral wall of the SMV or the SMPV confluence may not always be appreciated on preoperative imaging. Because of this, surgeons without the technical ability to perform venous resection may be in the position of being unable to separate the SMV from the tumor, predisposing to an incomplete resection or an inadvertent venotomy. Importantly, deformity of the vein wall at the tumor interface, even if subtle, often indicates tumor adherence. This may be appreciated only if the CT images are performed with a venous phase of contrast enhancement and are accurately interpreted.

5. When vascular resection and reconstruction is performed by experienced surgeons at institutions where such surgery is frequently performed, morbidity and mortality are not increased compared with standard PD without the need for vascular resection. ${ }^{5,8}$

Current recommendations for optimal clinical care and clinical research:

1. Surgeons who perform PD should carefully review the preoperative cross-sectional imaging studies and assess the relationship of the low-density tumor to the adjacent arteries (celiac, hepatic, SMA) and veins (SMV, PV). Aberrant hepatic arterial anatomy should be apparent on preoperative imaging and should not be an unexpected finding at the time of laparotomy. The relationship of the tumor to the SMPV confluence and the presence or absence of a deformity in the contour of the vein should alert the surgeon to the need for venous resection. The anatomy of the SMV as related to the location of the jejunal branch (with respect to the SMA) and the anatomy of the inferior mesenteric vein should also be identified on preoperative imaging.

2. Surgeons who perform PD should have a strategy to deal with unexpected venous adherence at the time of surgery.

3. When the tumor cannot be separated from the SMV, PV or SMPV confluence, a controlled venous resection and reconstruction should be performed. We usually use inflow occlusion on the SMA to prevent small bowel edema which may complicate the pancreatic and biliary reconstruction. ${ }^{8}$

4. The surgeon must document (in the operative dictation) the presence or absence of a complete gross resection at the time of $\mathrm{PD}$. The pathologist should examine the PD specimen in conformity with College of American Pathologists (CAP) or American Joint Committee on Cancer (AJCC) guidelines which include careful analysis of the SMA margin. Such margin analysis should be performed irrespective of whether or not vascular resection has been performed. The current (6th) edition of the AJCC Staging Manual emphasizes the importance of the $\mathrm{R}$ designation in all pathology reports and for this disease (and the operation of PD in particular) the operative dictation and the pathology report must be integrated to develop an accurate $\mathrm{R}$ designation. ${ }^{10}$ Manuscripts which assess 
vascular (usually venous) resection as a prognostic factor for survival must include such a system for the assessment of $\mathrm{R}$ status and their system must be accurately described in the "Methods" section of the manuscript. ${ }^{8}$

\section{REFERENCES}

1. Giovanni R, Paolo M, Niccolo P, et al. Does portal-superior mesenteric vein invasion still indicate irresectability for pancreatic carcinoma? Ann Surg Oncol. 2009. DOI:10.1245/s10434008-0281-8.

2. Fortner JG. Regional resection of cancer of the pancreas: a new surgical approach. Surgery. 1973;73:307-20.

3. Riall TS, Cameron JL, Lillemoe KD, Campbell KA, Sauter PK, Coleman J, et al. Pancreaticoduodenectomy with or without distal gastrectomy and extended retroperitoneal lymphadenectomy for periampullary adenocarcinoma-part 3: update on 5-year survival. J Gastrointest Surg. 2005;9:1191-204; discussion 204-6.

4. Pawlik TM, Abdalla EK, Barnett CC,, Ahmad SA, Cleary KR, Vauthey JN, et al. Feasibility of a randomized trial of extended lymphadenectomy for pancreatic cancer. Arch Surg. 2005;140:584-9; discussion 9-91.

5. Raut CP, Tseng JF, Sun CC, Wang H, Wolff RA, Crane CH, et al. Impact of resection status on pattern of failure and survival after pancreaticoduodenectomy for pancreatic adenocarcinoma. Ann Surg. 2007;246:52-60.

6. Allard WJ, Matera J, Miller MC, Repollet M, Connelly MC, Rao $\mathrm{C}$, et al. Tumor cells circulate in the peripheral blood of all major carcinomas but not in healthy subjects or patients with nonmalignant diseases. Clin Cancer Res. 2004;10:6897-6904.

7. Husemann Y, Geigl JB, Schubert F, Musiani P, Meyer M, Burghart E, et al. Systemic spread is an early step in breast cancer. Cancer Cell. 2008;13:58-68.

8. Tseng JF, Raut CP, Lee JE, Pisters PW, Vauthey JN, Abdalla EK, et al. Pancreaticoduodenectomy with vascular resection: margin status and survival duration. J Gastrointest Surg. 2004;8:935-49.

9. Katz MHG, Fleming JB, Pisters PWT, Lee JE, Evans DB. Anatomy of the superior mesenteric vein with special reference to the surgical management of first-order branch involvement at pancreaticoduodenectomy. Ann Surg. 2008;248(6):1098-102.

10. Exocrine pancreas. In: Greene FL, Page DL, Fleming ID, Fritz A, Balch CM, Haller DG, et al., editors. AJCC cancer staging manual. New York: Springer; 2002. p. 157-64. 